P155 (continued)

Funding: John Harvey Kellogg Endowment Research Funds, MSU NSF-AGEP.

\section{P156 A Comparison of the Nutrient Content Between Gluten-Free Foods to Matched Gluten- Containing Products}

CoraCoker, coracricket@gmail.com, University of Florida, 3038 McCarty Hall D, Gainesville, FL 32611; C. Dunn, MS, RD; K. Shelnutt, PhD, RD

Objective: To compare macronutrient and micronutrient content of gluten-free (GF) products from a variety of categories to matched gluten-containing (GC) products.

Design, Setting and Participants: Nutrition information for GF products $(n=104)$ was obtained from nutrition labels provided on GF product manufacturer websites. Nutrition information for equivalent matched GC products $(n=104)$ was obtained during on-site data collection at grocery stores in Gainesville, Florida. Products were matched and grouped based on product type, preparation, and description. Outcome Measures and Analysis: All products were standardized to 100 gram servings. Data were analyzed using Microsoft Excel. Researchers tested for significant differences in macronutrient and micronutrient content using student's t-test, with significance set at $\mathrm{p}<.05$.

Results: Percent Daily Value (\%DV) for thiamin, niacin, riboflavin, iron, and folate were significantly higher in GC loaf breads, oats/cereals, and baking mixes than in their GF counterparts $(\mathrm{p}<.02)$. However, the \%DV for calcium was significantly higher in GF versus GC cookies $(\mathrm{p}=.01)$. GC pastas had significantly higher protein content than GF pasta $(\mathrm{p}<.0001)$, while total fat for granola/ granola bars and loaf breads was significantly higher in GF products versus GC products $(\mathrm{p}<.02)$.

Conclusions and Implications: Significant differences in micro- or macronutrients were detected in all categories. These micronutrient differences are likely due to the enrichment and fortification of refined grain products. Following a GF diet may lead to altered patterns of nutrient intake, including an inadequate intake of certain micronutrients if a multivitamin is not also consumed. Nutrition education for individuals following a GF diet should include specific information about meeting micronutrient and macronutrient recommendations.

Funding: University of Florida/IFAS Dean for Research.

\section{P157 Understanding Why Women of Childbearing Age Follow a Gluten-Free Diet and Determining Where They Get Their Information}

Drew Carlton, drewcarlton1414@ufl.edu, University of Florida, 3038 McCarty Hall D, Gainesville, FL 32611; C. Dunn, MS, RD; K. Shelnutt, PhD, RD

Objective: To better understand why women of childbearing age follow a gluten-free (GF) diet and to determine where they get information on GF diets.
Design, Setting and Participants: A convenience sample $(n=86)$ of women of childbearing age $(18-45$ y) following a GF diet completed an online survey developed by the researchers that included demographic questions and evaluated reasons for starting a GF diet and where they obtained their information.

Outcome Measures and Analysis: Descriptive statistics from the online survey are provided.

Results: The majority of participants were white $(86 \%)$. Thirty-one percent were between 20-24 years, and 40\% were university students. When asked how long they had followed a GF diet, 23\% indicated 1-2 years while only $12 \%$ indicated more than five years. Participants indicated starting a GF diet for one or more of the following reasons: celiac disease diagnosis (44\%), non-celiac gluten sensitivity (49\%), irritable bowel syndrome (26\%), and wheat allergy (23\%). When presented with the statement, "A gluten free diet alone provides me with adequate amounts of vitamins and minerals," 64\% either agreed or strongly agreed. Only $14 \%$ reported consulting a Registered Dietitian to obtain information regarding their GF diet. Other sources included friends and family (58\%), medical websites (39\%), blogs (36\%), and magazines (10\%).

Conclusions and Implications: The women surveyed are not aware that their diet may be low in certain micronutrients if they do not consume a multivitamin/mineral supplement and are not seeking information from a qualified health professional. These women should be encouraged to consult a Registered Dietitian to obtain information on how to properly follow a GF diet.

Funding: University of Florida/IFAS Dean for Research.

\section{P158 Racial/Ethnic Differences in the Associations Between Parents' Body Mass Index, Behaviors and Child Weight Status}

Nurgul Fitzgerald, PhD, RD, nurgul.fitzgerald@rutgers.edu, Rutgers University, 26 Nichol Avenue, New Brunswick, NJ 08901; J. Theiss, PhD; J. Dewar, MS, University of Nevada Cooperative Extension; A. Wozniak, MEd, CHES;

S. Musaad, University of Illinois at Urbana-Champaign; A. Mobley, PhD, RD, University of Connecticut; M. SigmanGrant, PhD, $R D$, University of Nevada Cooperative Extension

Objective: Obesity is linked to racial/ethnic and socioeconomic disparities, but little is known about the potential intermediary role of parental behaviors. This study examined the racial/ethnic differences in parent BMI and parental behaviors as potential determinants of child weight status.

Design, Setting and Participants: Data were collected through interviews with 432 low-income mothers of preschool age children in a multi-state study. Body weight and height were measured.

Outcome Measures and Analysis: Healthy food intake and physical activity behavior checklist (BC), parental dietary modeling (PDM), parental overt and covert control on child's diet, caregiver feeding style (demanding; respon- 